\title{
Representing Spatial Location and Layout From Sparse Kinesthetic Contacts
}

\author{
Roberta L. Klatzky \\ Carnegie Mellon University
}

\author{
Susan J. Lederman \\ Queen's University
}

\begin{abstract}
Participants' fingers were guided to 2 locations on a table for $3 \mathrm{~s}$, then back to the start. They reported distances and angles between the locations by (a) replacing 1 or 2 fingers, (b) translating the contacted configuration, or (c) estimating distance or angle alone. Distance error increased across these conditions. Angular error increased when the angular reference axis was rotated before the response. Replacing 1 finger was impaired by a change in posture from exposure to test. The results suggest a kinesthetic representation is used to replace the fingers, but to estimate distance and angle at new locations, a configural representation is computed. This representation is oriented within an extrinsic reference frame and maintains shape more accurately than scale.
\end{abstract}

When people sit at a desk or table, the region of space within reach of their hands, called peripersonal or manipulatory space (Lederman, Klatzky, Collins, \& Wardell, 1987), typically holds a variety of tangibly distinct surfaces or objects. Often sparse touch, that is, contact with just one or two locations, is sufficient to determine the layout of objects and guide further reaching and manipulation. Consider a hypothetical scenario in which you put your coffee cup and pen down on your desktop. This action sets up a spatial representation that allows you to reach for each object again, without the need for vision. Additionally, you may know the spatial relations between the cup and pen, so that upon touching one you can move directly to the other.

This scenario illustrates two capabilities that may arise from touching a small number of points. One is knowing where the points are in space. The other is knowing the configuration or layout they form in terms of the metric properties of distance and angle between contacts. (By angle, we mean the bearing from one point to another, relative to a reference direction.) The parameters of distance and angle correspond to two essential components of a configuration-its scale and shape, respectively. The present studies assessed people's ability to return to touched locations and to report their layout, in order to determine the nature of the underlying representations that are formed from sparse contacts. In a series of experiments, participants' fingers were guided to locations in space for a few seconds, then back to a start position, whereupon they returned to the locations or reported metric prop-

Roberta L. Klatzky, Department of Psychology, Carnegie Mellon University; Susan J. Lederman, Department of Psychology, Queen's University, Kingston, Ontario, Canada.

This work was supported by National Eye Institute Grant EY 09740. We acknowledge the assistance of Jesse Kates and Andrew Thompson with data collection, and the very helpful comments of three anonymous reviewers.

Correspondence concerning this article should be addressed to Roberta L. Klatzky, Department of Psychology, Carnegie Mellon University, Pittsburgh, Pennsylvania 15213-3890. E-mail: klatzky@cmu.edu erties of the layout, subject to a translation or rotation. This task relied primarily on the kinesthetic component of touch, which uses inputs from the muscle, tendon, and joint receptors; the cutaneous input varies little (although there is evidence that fingertip pressure gradients contribute to the ability to point to previously touched locations; Rao \& Gordon, 2001).

Interest in the formation of spatial representations from sparse, kinesthetically sensed contacts has been motivated both from a basic research perspective, as reviewed below, and more recently, by the emergence of force-feedback devices that create virtual haptic environments. The most widely used of these is the commercially available PHANTOM (SensAble Technologies, Woburn, MA). In a common configuration, the user inserts the index finger into a thimble-shaped connector; in a dual-device system, the index fingers of the left and right hand are inserted. The user can move each finger with six degrees of freedom while receiving forces (usually along three orthogonal axes) from simulated contact. The forces delivered to each finger simulate, however, only a single-point contact with the environment at any one time. Under these circumstances, people can extract size and shape features of a virtual display to at least a coarse degree (e.g., O'Malley \& Goldfarb, 2002), indicating that a series of exposures to one or two contact positions has considerable perceptual utility. Force-feedback devices can also be used in a guidance mode, in which users are led over the shapes of target patterns, as in learning to draw Japanese characters (Solis, Avizzano, \& Bergamasco, 2002). Studies of kinesthetically induced representations potentially have implications for the utility of such devices.

\section{Spatial Representation}

Following Klatzky (1998), we define a spatial representation as a set of parameter values that describes the locations of points or regions in space, where the parameters are defined with respect to some reference system. One issue that has been addressed in previous research on representations of manipulatory space is: What type of reference system or systems are used to locate contact points? A related issue pursued here is: Given that a 
representation has been formed from kinesthetic input to provide a parametric description of the input, what additional parameters can be derived, and how well? Our underlying assumption was that the process of computing new parameters, which is equivalent to forming a new representation, would be subject to systematic error and loss of precision (noise). This means that parameters that are conveyed directly by a representation would be reported more accurately than parameters that must be derived by second-order processes.

Indeed, it has been demonstrated that properties that seem logically capable of derivation from a representation are not necessarily capable of being processed accurately. In particular, knowledge of the locations of touched points does not guarantee that people have access to metric relations among them. For example, when an observer views spatial locations within $25 \mathrm{~m}$ or so (ambulatory space), he or she may have knowledge of the egocentric locations - as evidenced by the ability to walk to them without vision from the point of observation-but may show substantial error in computing the exocentric distances between two locations (Loomis, Da Silva, Fujita, \& Fukusima, 1992). A similar effect is found in manipulatory space (i.e., for locations within $50 \mathrm{~cm}$ ), under monocular viewing conditions (Loomis \& Philbeck, 1994). As we report below, considerable errors also occur when people derive both angle and distance estimates from haptically explored configurations.

\section{Representations in Manipulatory Space}

Several general classes of spatial representations for manipulatory space can be distinguished in terms of the underlying reference system and the parameters it provides. We refer to them here as kinesthetic, extrinsic (egocentric or exocentric), and configural. In addition to these spatial reference systems, it is possible that people use relatively weak heuristics for coding spatial properties, which they base on the temporal or effortful properties of movement.

\section{Kinesthetic}

A kinesthetic representation of haptically perceived locations provides a code in terms of sensory inputs from muscles, tendons, or joints (e.g., the joint angles used to reach a point in space), not in terms of external space. This form of representation is necessarily egocentric; that is, the parameters of spatial locations are referred to the body. Given the many-to-one mapping from joint angles to spatial locations, the kinesthetic representation of a touched location is likely to be transient. The presence of a kinesthetic code allows people to return to previously felt positions in space but may not directly convey the distance or angle between them. Kinesthetic coding predicts that performance would be impaired in location or movement-reproduction tasks in which there is a change between exposure and test with respect to muscle, tendon, or joint position. Tests of this prediction have found that performance is sometimes, but not consistently, degraded by a change in limb or body posture (for degradation, see, e.g., HelmsTillery, Flanders, \& Soechting, 1994; Wallace, 1977; for null effects see Baud-Bovy \& Viviani, 1998; Larish \& Stelmach, 1982; Rao \& Gordon, 2001; Wallace, 1977).

\section{Extrinsic}

A haptic spatial representation that is extrinsic makes reference to locations in external space, not to the exploratory pattern that was used to feel those locations. An extrinsic representation can be egocentric; for example, a major axis is aligned in external space with the body's frontal plane or the origin is located on the body. Alternatively, it can be exocentric (sometimes called allocentric); that is, the parameters are defined externally to the body (e.g., a major axis is aligned with the table edge). Millar $(1976,1994)$ has suggested preferential use of a body-centered reference (particularly the sagittal axis) for locating multiple touched points. Like kinesthetic coding, use of an extrinsic spatial code (egocentric or exocentric) would allow people to return to previously contacted positions in space but would not necessarily support computation of metric properties. Evidence favoring extrinsic over kinesthetic coding is found in studies cited above comparing location reproduction with or without postural changes and finding null effects (Baud-Bovy \& Viviani, 1998; Larish \& Stelmach, 1982; Rao \& Gordon, 2001; Wallace, 1977). It has also been found that people produce the endpoints of movements better than the movements themselves (Jaric, Corcos, Gottlieb, Illis, \& Latash, 1994; see also Smyth, 1984, for a review); however, this outcome is ambiguous, as endpoint reproduction could be based on kinesthetic memory for final postures rather than an extrinsic location code (Rosenbaum, Meulenbroek, \& Vaughan, 1999).

\section{Configural}

A configural representation locates salient points or landmarks of a layout in relative, rather than absolute, terms by indicating angles and distances between them. The angles indicate the layout's shape, and the distances its scale. This type of representation does not directly provide the necessary information to return to the touched locations. As defined, it is object-centered, in which case the parameters describing the layout do not change if it is translated or rotated. Klatzky (1999) tested for use of a configural representation in a task in which participants followed a twosegment raised line with an index finger and then attempted to return directly to the starting point, thereby completing a triangle. The response distance and angle relative to the second outbound segment were measured. In some conditions, between the outbound segments and the return, the entire path was to be mentally translated along a line parallel to the front of the body. This caused only a small increase in distance error and no increase in angle error, suggesting a location-independent representation of the path configuration. However, when participants had to mentally rotate the path before making the return response, there was a profound increase in angle error. The rotation effect suggests that the configural representation was oriented with respect to the participant's body or an external reference axis, although it was not tied to a fixed location in space.

\section{Movement-Based Heuristics}

Another possibility for computing metric properties from haptic exposure without relying on a location representation is to use the pattern of movement through space as a cue, albeit an inexact one A variety of evidence suggests that distance estimation relies to 
some extent on such ad hoc heuristics, which can be a source of sizable systematic error. For example, Brambring (1976) found that participants who felt two sides of a right triangle and then estimated the length of the third side evidenced use of a "cityblock metric", implying that their distance judgments reflected the path they traveled. Haptically based distance estimates have also been found to depend on the direction of a movement and its speed (e.g., Armstrong \& Marks, 1999). Lederman, Klatzky, and Barber (1985) found that people's estimates of the Euclidean distance between the endpoints of a touched curved path increased with the path length, with up to a 2:1 error. This effect was present even when the participants held one finger at the starting point while moving the other along the path, in which case the end-to-end distance was momentarily directly tangible. Moreover, the movement-induced distortion was greater at slower speeds (Lederman et al., 1987). Fasse, Hogan, Kay, and Mussa-Ivaldi (2000) found that perceived lengths of objects that were felt using a robotic manipulandum showed systematic distortions that were similar to errors in motor production on the order of $25 \%$. Angle reports were similarly distorted, on the order of $30 \%$, but could not be predicted from the length errors.

\section{Relations Among the Representations}

The literature reviewed above points to the availability of multiple mechanisms for representing manipulatory space, depending somewhat on the task constraints. Accordingly, the question is not so much what is the sole operative representation, but rather which representations are invoked and when. We propose a relationship among representations as shown in Figure 1. To begin with, the cues that differentiate contact points in space arise primarily in kinesthetic receptors in muscles, tendons, and joints. These cues give rise to a representation of the posture at the time of contact. Whereas the parameters of this kinesthetic representation support returning to the touched locations, such a representation does not directly convey metric relations between touched points or between those points and reference elements in the external space. For these purposes, configural and extrinsic representations, respectively, must be derived. The configural representation may be formed directly from kinesthetic cues, or an extrinsic representation of touched locations may be an intermediate stage. The converse, deriving the extrinsic representation from an objectcentered configural code, is not possible. Each succeeding level of representation requires a process of computation that is subject to error.

Some tasks require coordinating representations. For example, someone might be required to report the angle between touched locations relative to an external reference axis, which would require that a configural representation be oriented within an extrinsic frame. Finally, we assumed that ad hoc heuristics would be applied when direct readouts or algorithmic computations from other representations fail.

\section{The Present Approach}

The goal of the present studies was to test the general ordering in Figure 1 by devising tasks for which different levels of representation would be needed and by then comparing the level of error. As was noted above, the underlying assumption is that

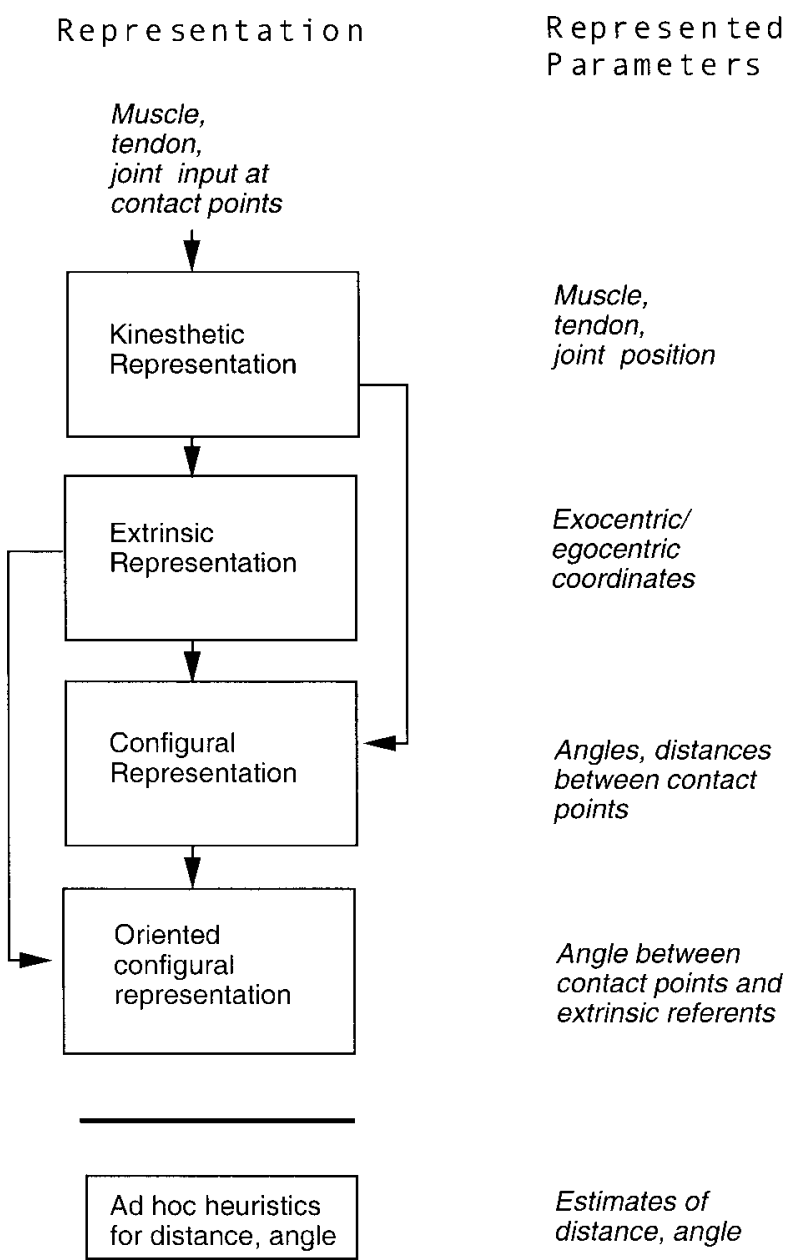

Figure 1. Proposed relations among spatial representations for kinesthetic contacts. Left column shows levels of representation, with possible successive computations connected by arrows. Right column shows parameters directly conveyed by the representation at left.

extracting parameters directly from a representation will lead to lower error than computing a new level of representation that provides those parameters. Estimation from a heuristic process should be particularly error prone.

Experiment 1 provided a direct comparison between the accuracy with which people replace their two index fingers at previously touched sites and their accuracy in estimating the interpoint distances. Whereas a kinesthetic representation should support replacement, estimating the interpoint distance should require deriving a configural representation, which would increase error. This study also incorporated a single-finger replacement task to further test for kinesthetic coding. Experiment 2 added angle estimation to the repertoire of tasks used in Experiment 1, using the same underlying logic. Experiment 3 directly assessed the efficacy of a configural representation by having participants reproduce the entire layout in a new location and measuring angle and distance errors. Finally, following the study of Klatzky (1999), Experiment 4 required participants to reproduce the layout after a mental rotation, to investigate whether the configural representa- 
tion was tied to an extrinsic reference orientation. If so, the rotation demand would require further computation or heuristic processing, adding error.

\section{Experiment 1: Full or Partial Finger Replacement Versus Distance Estimation}

In this study, experimenters guided participants' fingers to two target locations in space, and participants then either replaced one or both fingers at their original locations or estimated distance in a new location. The experiment was intended to address whether replacing the fingers relies directly on a kinesthetic representation, whereas estimating distance requires deriving an additional representation. We anticipated that error in distance estimation would be greater than the distance error found when replacing the fingers at the target locations because of the necessity of forming a new representation that conveys metric properties, as shown in Figure 1. When a single finger was replaced, the participant was sometimes required to change the limb between presentation and test. Further evidence for a kinesthetic representation underlying the finger-replacement task would be found if a change in the responding hand increased replacement error.

\section{Method}

Participants. Ten university students participated for course credit, 7 men and 3 women. Six were right handed, and 3 were left handed, as indicated by self-report; the handedness of 1 participant was not ascertained.

Apparatus and procedure. A digitizing tablet (Calcomp 2300; Calcomp, Anaheim, CA), measuring $68 \mathrm{~cm} \times 57 \mathrm{~cm}$ and running with a resolution of $1 \mathrm{~mm}$ over a programmable area of $61 \mathrm{~cm} \times 46 \mathrm{~cm}$, was placed on the table so that its front edge was $2.5 \mathrm{~cm}$ from the edge of the table. Each participant sat at the table aligned with the edge, near enough to be able to reach the tablet's far edge comfortably. On each trial, the experimenter placed the participant's index fingers of the right and left hand so they were contiguous at a start location near the participant. From that location, the experimenter simultaneously guided the fingers to two target locations on the tablet, released them and waited $3 \mathrm{~s}$, and then guided them back to the start location. The fingers were not overtly moved during the 3-s exposure interval. At that point, the participant made one of the following four responses, as designated by the experimenter:

Replace (two finger): The participant moved the right and left index fingers back to the target locations, simultaneously or successively as desired. (Generally, these responses were made smoothly.)

Replace (same side-one finger): The participant moved the right or left index finger back to its target location, as designated by the experimenter.

Replace (opposite side-one finger): The participant moved the right index finger to the target location of the left finger, or vice versa, as designated by the experimenter.

Distance estimation: At the start location, the participant estimated the distance between the previous locations of the right and left fingers, by moving one finger (designated by the experimenter as right or left on a random basis) until the distance between them matched the target distance.

The positions of the fingers at the point of response were recorded by the experimenter, who placed a stylus under the center of the fingertip and depressed it. The position of the stylus on the digitizer was stored in the computer.

The target locations fell within six regions, arranged around the center of the digitizer pad in two rows of three each, as shown in Figure 2B. Within each target region, four pairs of target locations were defined, as illustrated in Figure 2A. The two locations in a pair were centered on the midpoint of the target region and separated by a total distance of $8,16,24$, or $32 \mathrm{~cm}$. These values were chosen to represent reasonable parametric variation, to be within comfortable reach, and to constrain the most eccentric locations to within $8 \mathrm{~cm}$ of the outer edge of the digitizer pad, thus avoiding responses that would fall outside the measurable area. The angle from the left member of the target pair to the right member, was $-15^{\circ},-10^{\circ},-5^{\circ}$, $5^{\circ}, 10^{\circ}$, or $15^{\circ}$ (positive $=$ counterclockwise) relative to the horizontal. As angle judgments were not the focus of this study, these values were intended to provide some variation from the horizontal and to constrain the targets nearest the participant to at least $8 \mathrm{~cm}$ forward of the start locations.

There were also six possible start locations, where the fingers rested before moving to the targets, shown in Figure 2B. A particular start location was yoked to each target region so that all target locations within a region were reached from the same starting point. (Specifically, for starting positions $1-6$, associated regions were far center, near left, near center, near right, far left, and far right, respectively.)

\section{A. Target locations around a region center}

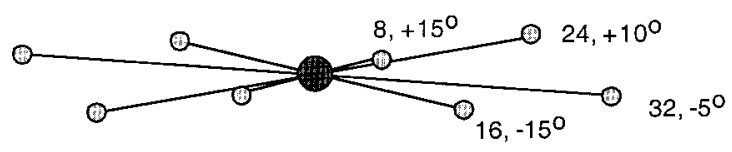

\section{B. Layout of regions and start locations}

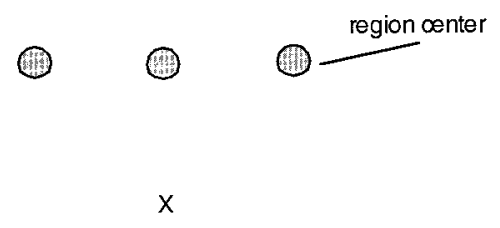

1

2

3

4

5

6

\section{$\overline{50 \mathrm{~mm}}$ table edge}

Figure 2. A: For Experiment 1, a possible set of target locations centered in a region; each pair is marked at right with the corresponding intertarget distance $(\mathrm{cm})$ and angle (degrees). B: Layout of the regions and start locations in Experiment 1. 
Table 1

Definitions and Applicable Instruction Conditions for Each Error Measure

\begin{tabular}{|c|c|c|}
\hline Error measure & Definition & Applicable instruction conditions \\
\hline \multirow[t]{3}{*}{ Location } & \multirow[t]{3}{*}{ Distance between response locations (RLs) and target locations (TLs) } & Replace (two finger) \\
\hline & & Replace (same side-one finger) \\
\hline & & Replace (opposite side-one finger) \\
\hline \multirow[t]{3}{*}{ Interfinger distance, signed } & \multirow[t]{3}{*}{ (Distance between RLs) - (Distance between TLs) } & Replace (two finger) \\
\hline & & Translate \\
\hline & & Distance estimation \\
\hline \multirow[t]{3}{*}{ Interfinger distance, absolute } & \multirow[t]{3}{*}{$\mid($ Distance between RLs) - (Distance between TLs) $\mid$} & Replace (two finger) \\
\hline & & Translate \\
\hline & & Distance estimation \\
\hline \multirow[t]{3}{*}{ Interfinger angle, signed } & \multirow[t]{3}{*}{ (Angle between RLs) - (Angle between TLs) } & Replace (two finger) \\
\hline & & Translate \\
\hline & & Angle estimation \\
\hline \multirow[t]{3}{*}{ Interfinger angle, absolute } & \multirow[t]{3}{*}{$\mid$ (Angle between RLs) - (Angle between TLs) $\mid$} & Replace (two finger) \\
\hline & & Translate \\
\hline & & Angle estimation \\
\hline
\end{tabular}

All four distances were represented by target-location pairs at each region, and angles were sampled so that within each distance, each angle occurred once within each region. The combination of six regions, four intertarget distances, and four instructional conditions yielded 96 trials, which were administered in random order. The participants were not told which response would be required until their hands had returned to the start location from the target locations.

\section{Results}

Five error measures were computed from the data. Table 1 defines each and indicates which measures were applicable with each instruction. One measure was location error, the Euclidean distance from a response location to the corresponding target. The remaining measures could be computed as signed or absolute error. Whereas signed errors indicated systematic trends that were directionally consistent across participants, absolute errors incorporated not only this systematicity but errors that varied in direction from one observation to another (i.e., noise). One measure was interfinger distance error, or how discrepant the report was about the distance between members of a target pair, regardless of their absolute location. Another was interfinger angle error, or how discrepant the report was about the angle between members of a target pair, regardless of their absolute location. Table 2 reports the various error measures for this and subsequent experiments, averaged over geometric parameters of the layout (target distance, target angle, and region).

Location error. This measure could be defined for the three replace instructions: two finger, same side-one finger, and opposite side-one finger. The centroids of the response locations of the fingers, which produce the location error, are shown by region and instruction in Figure 3. An analysis of variance (ANOVA) on the location error, with factors of instruction (three); region (six); and distance (four), showed effects of instruction, $F(2,18)=16.34$, $p<.01$; region, $F(5,45)=6.92, p<.01$; and distance, $F(3,27)=$ $13.06, p<.01$. None of the interactions approached significance. The effect of instruction indicates that accuracy decreased from the two finger, to the same side-one finger, to the opposite side-one finger response. The effect of region reflects an advantage for regions nearer the body. A similar advantage for proprioceptive localization near the body was found by van Beers, Sittig, and

Table 2

Error Measures (Location, Absolute and Signed Interfinger Distance, Absolute and Signed Interfinger Angle) by Relevant Instruction (Replace, Translate, Estimation) for All Experiments, Averaged Over Geometric Parameters of the Display

\begin{tabular}{|c|c|c|c|c|c|c|c|}
\hline \multirow[b]{2}{*}{ Error and experiment } & \multirow{2}{*}{$\begin{array}{c}\text { Replace } \\
\text { location }(\mathrm{mm})\end{array}$} & \multicolumn{3}{|c|}{ Interfinger distance $(\mathrm{mm})$} & \multicolumn{3}{|c|}{ Interfinger angle (deg) } \\
\hline & & Replace & Translate & Estimation & Replace & Translate & Estimation \\
\hline \multicolumn{8}{|l|}{ Absolute error } \\
\hline Experiment 1 & 28.7 & 27.8 & & 51.4 & 6.9 & & \\
\hline Experiment 2 & 26.7 & 24.3 & & 47.2 & 8.6 & & 11.4 \\
\hline Experiment 3 & 26.8 & 20.0 & 31.6 & 43.3 & 9.2 & 12.3 & \\
\hline Experiment $4(0 / 0)$ & & & 28.7 & & & 10.3 & \\
\hline \multicolumn{8}{|l|}{ Signed error } \\
\hline Experiment 1 & & 8.0 & & -28.9 & 2.8 & & \\
\hline Experiment 2 & & 13.8 & & 36.3 & 0.8 & & -0.7 \\
\hline Experiment 3 & & 4.4 & 16.4 & 27.0 & -0.2 & -0.1 & \\
\hline Experiment $4(0 / 0)$ & & & 16.7 & & & 1.4 & \\
\hline
\end{tabular}

Note. The replace instruction refers to the two-finger response. The geometric parameters of the display were as follows: Experiment 1, mean interfinger distance $=20 \mathrm{~cm}$, angle range $= \pm 15^{\circ}$; Experiments $2-4$, mean interfinger distance $=15 \mathrm{~cm}$, angle range $= \pm 45^{\circ}$. deg $=$ degrees. 


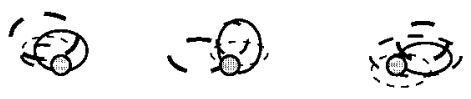

\section{Two Finger}

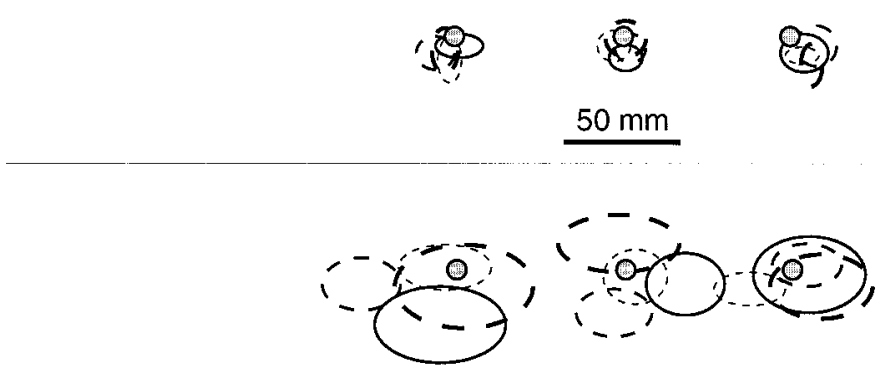

\section{Same Side One Finger}

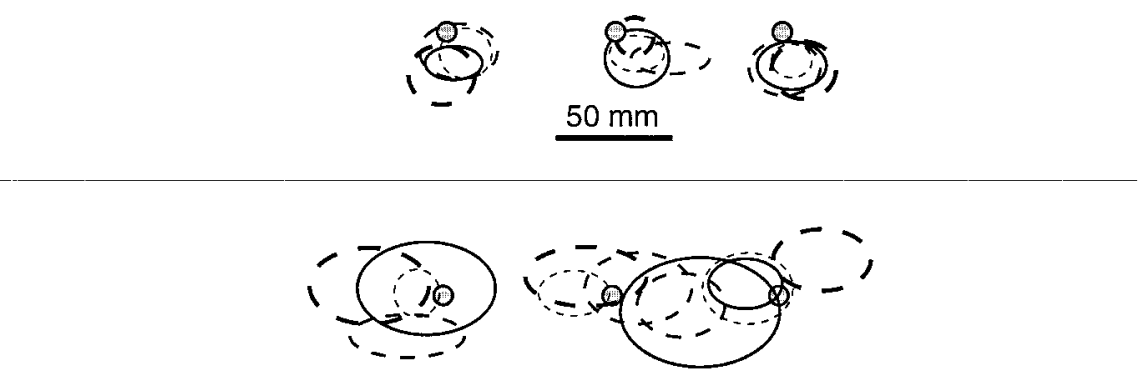

\section{Opposite Side One Finger}
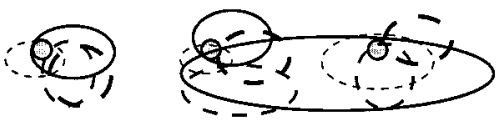

$50 \mathrm{~mm}$

Figure 3. Responses in the three replace instruction conditions of Experiment 1. Each panel shows the centers of the six target regions as dark circles. The four rings correspond to the four target pairs in the region. Rings composed of fine dashes, medium dashes, large dashes, and solid boundaries correspond to intertarget distances of $8,16,24$, and $32 \mathrm{~cm}$, respectively. We designated the center of the region as having coordinates $x_{\mathrm{c}}, y_{\mathrm{c}}$ and the average signed error in the $x$ and $y$ directions (averaging over participants and both left- and right-finger target locations) as $x_{\mathrm{e}}, y_{\mathrm{e}}$; thus, the center of a given ring is $x_{\mathrm{c}}+x_{\mathrm{e}}, y_{\mathrm{c}}+y_{\mathrm{e}}$. The center of the ring shows the average signed error in the $x$ and $y$ directions, treating the dark circle at the center of the region as the target location. The width and the height of the ring represent the sample standard deviation of the errors in the $x$ and $y$ directions, respectively. 
Denier van der Gon (1998). (Although region was confounded with starting location in our design, the systematic results with respect to the location of the target regions suggest that target and not starting location is what underlies the effect.) Finally, the effect of distance indicates a tendency for location replacement to show more error as the distance between the fingers increases.

Interfinger distance error. Figure 4 shows the mean and standard error of the absolute interfinger distance error for the two applicable instructional conditions, replace (two finger) and distance estimation, by distance (four) and region (six). Figure 5 shows the corresponding signed interfinger distance error. The ANOVA on absolute error showed effects of instruction, $F(1,9)=$ $24.58, p<.01$; overall error was higher by close to a factor of 2 for distance-estimation instructions $(51.4 \mathrm{~mm})$ over replace (two finger; $27.8 \mathrm{~mm})$. There was an effect of distance, $F(3,27)=$ 14.14, $p<.01$, and Instruction $\times$ Distance, $F(3,27)=5.17, p<$ .01: Error increased with distance, far more so for the distanceestimation condition, so that the greater error for distance estimation relative to replace (two finger) increased with distance. The three-way Instruction $\times$ Distance $\times$ Region interaction, $F(15$, $135)=2.02, p<.05$, is not easily interpreted. However, it does not contradict the general pattern of an advantage for replace instructions over estimation, more so at greater interfinger distances.

The corresponding ANOVA on signed interfinger distance error (Figure 5) showed effects of instruction, $F(1,9)=11.47, p<.01$; distance, $F(53,27)=51.94, p<.01$; and the following interactions: Instruction $\times$ Region, $F(5,45)=6.36, p<.01$; Instruction $\times$ Distance, $F(3,27)=34.73, p<.01$; and Instruction $\times$ Region $\times$ Distance, $F(15,135)=2.41, p<.01$. Both instructions showed a tendency for error to change from modest overestimation to increasing underestimation as distance increased, but the trend was much larger for distance estimation. Both instructions also showed a tendency for region effects to be greater at the longer distances, but the direction of these effects was opposite, leading to the three-way interaction.

Interfinger angle error. Angle errors were only calculable in the replace (two finger) condition. Figure 6 shows the absolute error by region and target distance and the signed error by region and target angle. Plotting the two measures against different variables captures the fact that absolute error was systematically related to distance (and not angle) and signed error was systematically related to angle (and not distance).

The ANOVAs on both types of interfinger angle error had region and distance as factors. The absolute error showed a significant region effect, $F(5,45)=4.59, p<.01$, reflecting greater accuracy in the front locations. There was also an effect of distance, $F(3,27)=12.83, p<.01$, due to a tendency for the absolute angle error to decrease as the target distance increased. However, this is likely to reflect the fact that any error in measuring the exact location of the fingers leads to greater error in the response angle as the fingers move closer together; in other words, the effect can be attributed to measurement artifact. The signed interfinger angle error showed only a significant Region $\times$ Distance effect, $F(15$ $135)=4.42, p<.01$; the main effects did not approach significance. As can be seen in Figure 6 (right), this interaction reflects the fact that signed interfinger angle errors were strongly related to target angle. Given that a positive sign denotes a counterclockwise rotation, the data show that participants tended to respond with a steeper (more vertically oriented) angle than the target angle, particularly when the target angle was positive. This effect is reminiscent of anchor effects found in other experiments with haptic (Lederman et al., 1985; Lederman \& Taylor, 1969) and visual (e.g., Huttenlocher, Hedges, \& Duncan, 1991) displays,
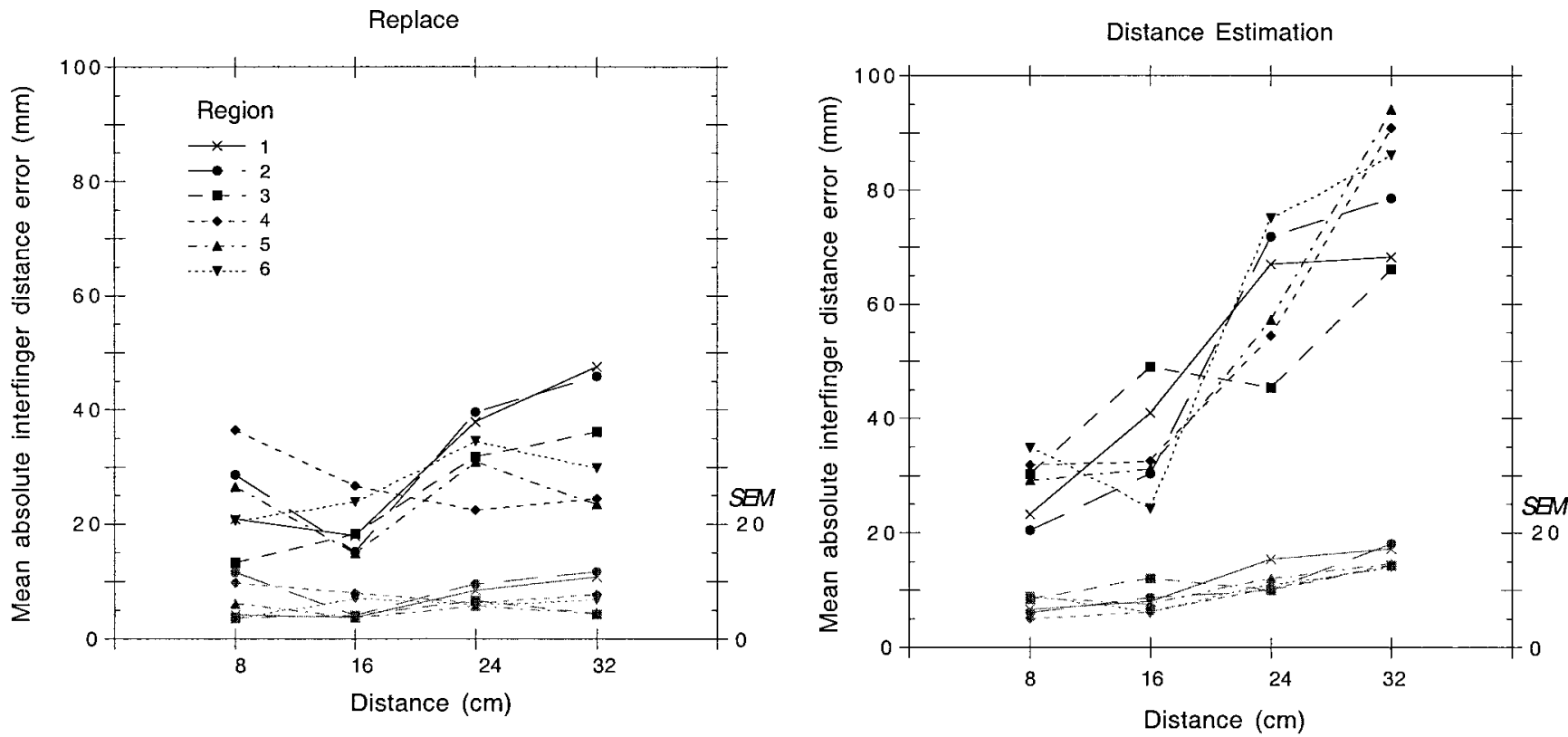

Figure 4. Mean and standard error of the absolute interfinger distance error for the replace (two finger) and distance-estimation instructions in Experiment 1 by target distance (four) and region (six). Regions are numbered left to right, far to near. 
Replace

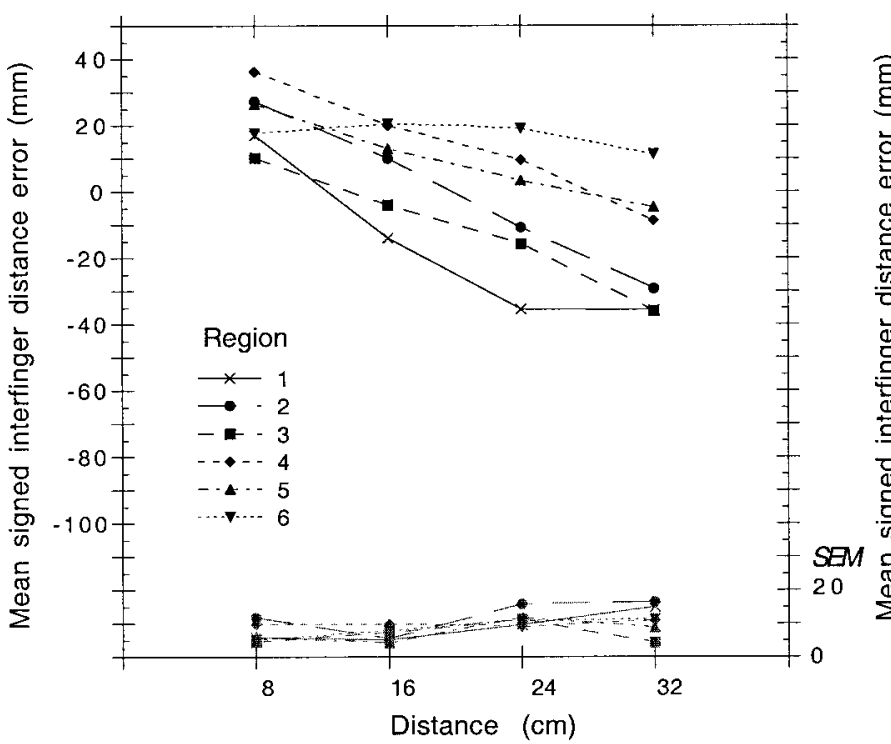

Distance Estimation

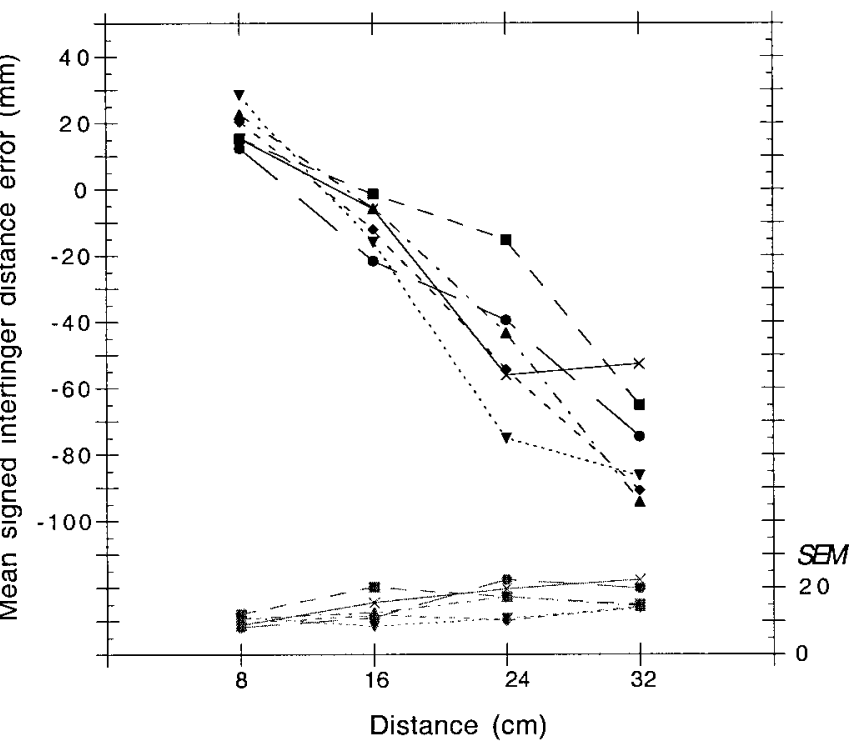

Figure 5. Mean and standard error of the signed interfinger distance error for the replace (two finger) and distance-estimation instructions in Experiment 1 by target distance (four) and region (six).

such that responses were drawn toward prototypical spatial locations.

\section{Discussion}

In general, the results are consistent with the proposal that participants' ability to reproduce contacted locations in space would rely on a kinesthetic representation. Accuracy in replacing the fingers at the target locations, as measured by distance of the touched locations from the targets, was greatest when both fingers were replaced, declined when only one was replaced, and declined further when the right finger moved to the left one's location or vice versa. The finding that performance was worst when one hand moved to the prior location of the other supports the assumption that a kinesthetic trace was used to report the touched location. The finding that replacing two fingers was better than replacing one on
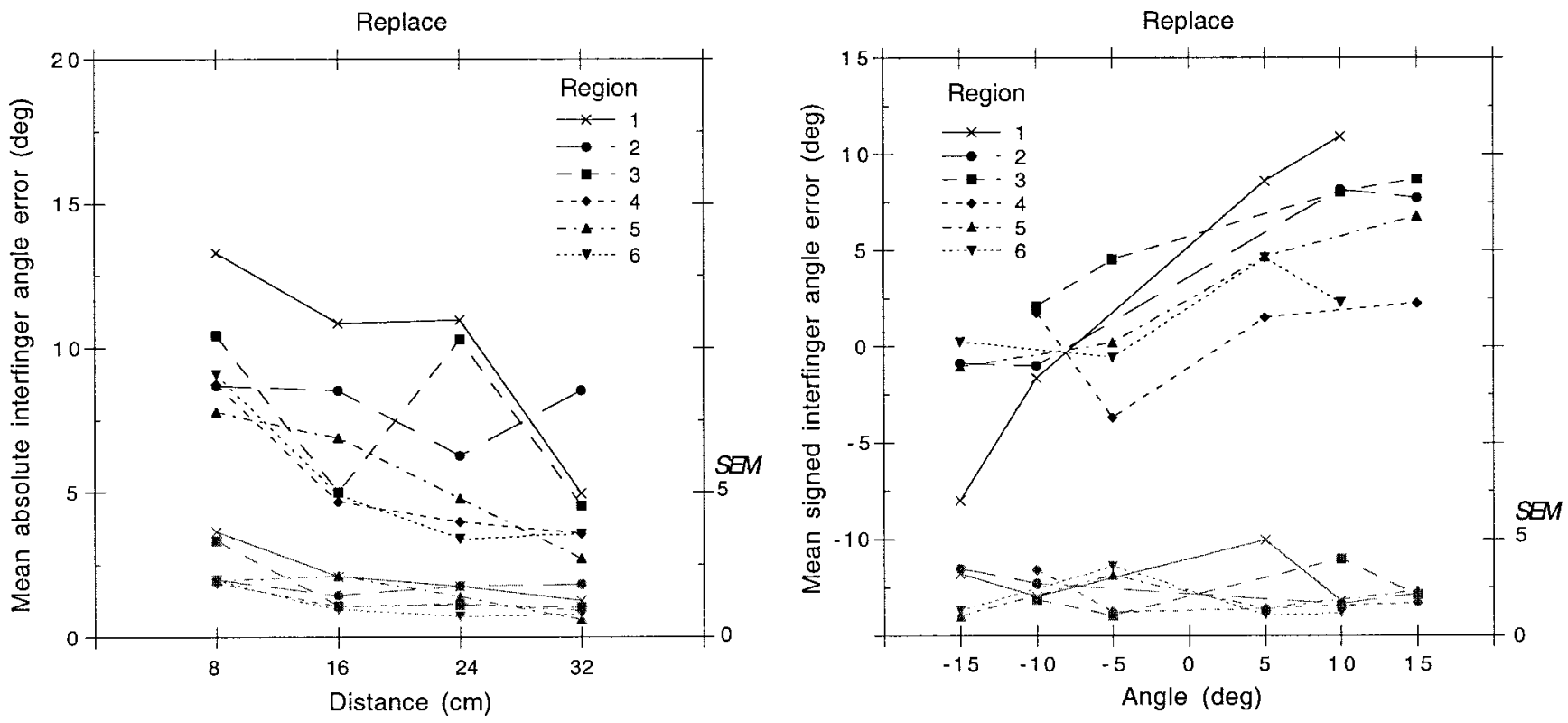

Figure 6. Left: Mean absolute interfinger angle error in Experiment 1 and the standard error of the mean by region and target distance. Right: Mean signed interfinger angle error in Experiment 1 and the standard error of the mean by region and target angle. deg $=$ degrees. 
its original side further suggests that the target locations were represented with respect to the full upper body position.

The interfinger distance errors indicate the effect of computing distance as a metric property of the layout, as depicted in Figure 1. The distance between the fingers was replicated far more accurately when participants replaced their fingers at the target than when they responded by estimating the distance at the edge of the tablet. The disadvantage for the distance-estimation response increased substantially with the actual interfinger distance, and the two responses-replacing the two fingers versus estimating the distance - showed different effects of target region, supporting the assumption that they relied on different memory representations.

The angle errors obtained when the two fingers were replaced averaged within $15^{\circ}$ of the target, and they largely resulted from a tendency to shift the fingers toward the vertical. Because angle estimation was not performed in Experiment 1, it does not allow a direct comparison between estimation and replacement for angles. This was done in the next experiment.

\section{Experiment 2: Distance or Angle Estimation Versus Replacing the Fingers}

The purpose of Experiment 2 was to contrast replacement of the two fingers on the target locations with explicit estimates of both distance and angle. The framework in Figure 1 suggests that replacement makes use of a kinesthetic representation, whereas estimation requires computation of a configural representation. In general, when distance and angle are reported by replacing the fingers, one would expect the levels of accuracy for the two measures to covary, because conditions that impair the kinesthetic representation (e.g., increasing the retention interval) should affect both components of the response. However, when distance and angle are reported as abstracted properties of a touched layout, one would not necessarily expect to find them strongly coupled, because different processes or heuristics could be used to derive them. In fact, a number of findings have indicated that these properties of haptic stimuli produce different levels of error and are affected by different variables. Fasse et al. (2000) found that distortions in perceived angle were not predictable from length errors. Klatzky (1999) showed that distance and angle reports were affected by translation and rotation of a felt triangle, respectively. Lederman et al. (1985) found that irrelevant movement between endpoints of a path led to systematic error in reports of Euclidean distance but not of angle.

We used a factorial design to manipulate distance and angle, and we increased the range of angles from $\pm 15^{\circ}$ in Experiment 1 to $\pm 45^{\circ}$ in Experiment 2 . The instructions to the participants introduced the frontal axis of the body as a reference for reporting the angle between the fingers. We expected to replicate the substantial difference between replace and estimation instructions for distance. A corresponding difference between the two responses in angle judgments would indicate a similar cost of computing the metric property from the kinesthetic representation that supports replacement.

\section{Method}

Participants. The participants were 10 university students who took part for course credit. There were 7 women and 3 men; all were right handed, as indicated by self-report. None had participated in Experiment 1 .

Layout of starting and target locations. There were two target regions, each centered along the sagittal axis of the participant's body, at distances of $18 \mathrm{~cm}$ and $41 \mathrm{~cm}$ from the tablet edge. The target pair members were separated by $6,12,18$, or $24 \mathrm{~cm}$ and formed an angle relative to the horizontal of $-45^{\circ},-15^{\circ}, 15^{\circ}$, or $45^{\circ}$. The combination of 4 intertarget angles $\times 4$ intertarget distances $\times 2$ target regions produced 32 target pairs, each of which occurred in conjunction with three responses for a total of 96 trials. One of two starting locations was assigned to each trial randomly, with the constraint that both were used equally often. The starting locations placed the two fingers $12 \mathrm{~cm}$ apart, symmetric about the sagittal plane, and at one of two distances inward from the tablet edge-22 $\mathrm{cm}$ or $35 \mathrm{~cm}$.

Procedure. As before, the participant's index fingers were guided from start locations to two target locations, and after $3 \mathrm{~s}$ the fingers were returned to the start locations. From this position, the experimental instructions were of the following three types:

Replace: The participant moved the right and left fingers to their previous locations. (In this and subsequent experiments, only twofinger replacement is involved, and the label replace is used to describe this condition.)

Angle estimation: At the start location, the participant moved the fingers vertically—simultaneously or separately, up or down—so as to replicate the angle formed between the two target locations relative to the frontal axis.

Distance estimation: At the start location, the participant moved the fingers horizontally — simultaneously or separately, right or left—so as to replicate the Euclidean distance formed between the two target locations.

\section{Results}

As before, there were three measures: location error (replace instruction only), interfinger distance error (replace and distanceestimation instructions), and interfinger angle error (replace and angle-estimation instructions). Figure 7 shows the signed interfinger distance error and interfinger angle error by region, distance, and angle for each instruction. Table 3 shows the absolute errors (averaged over region, as effects were minimal).

Location error. Two extreme values (more than two times larger than the next highest observed value) were eliminated and replaced with the maximum observed value of all participants. Unlike Experiment 1 (in which there were six regions and three instructions using replacement, in comparison with the present use of two central regions and only the two-finger replacement), none of the effects in the ANOVA on distance, angle, and region approached significance. The mean error was $26.7 \mathrm{~mm}$, similar to the corresponding value in Experiment 1 (see Table 2).

Interfinger distance error. The ANOVA on absolute interfinger distance error showed a significant effect of instruction, $F(1$, $9)=25.87, p<.01$, and an Instruction $\times$ Angle interaction, $F(3$, $27)=6.47, p<.01$, due to the fact that the instruction effect was somewhat greater for the wide angles $\left( \pm 45^{\circ}\right)$. As in Experiment 1, absolute distance error was almost twice as great for the distanceestimation instruction as for the replace instruction. The absolute error showed a main effect of angle, $F(3,27)=4.05, p<.05$, with higher error for the $\pm 45^{\circ}$ angles, and of Region $\times$ Angle, $F(3$, 

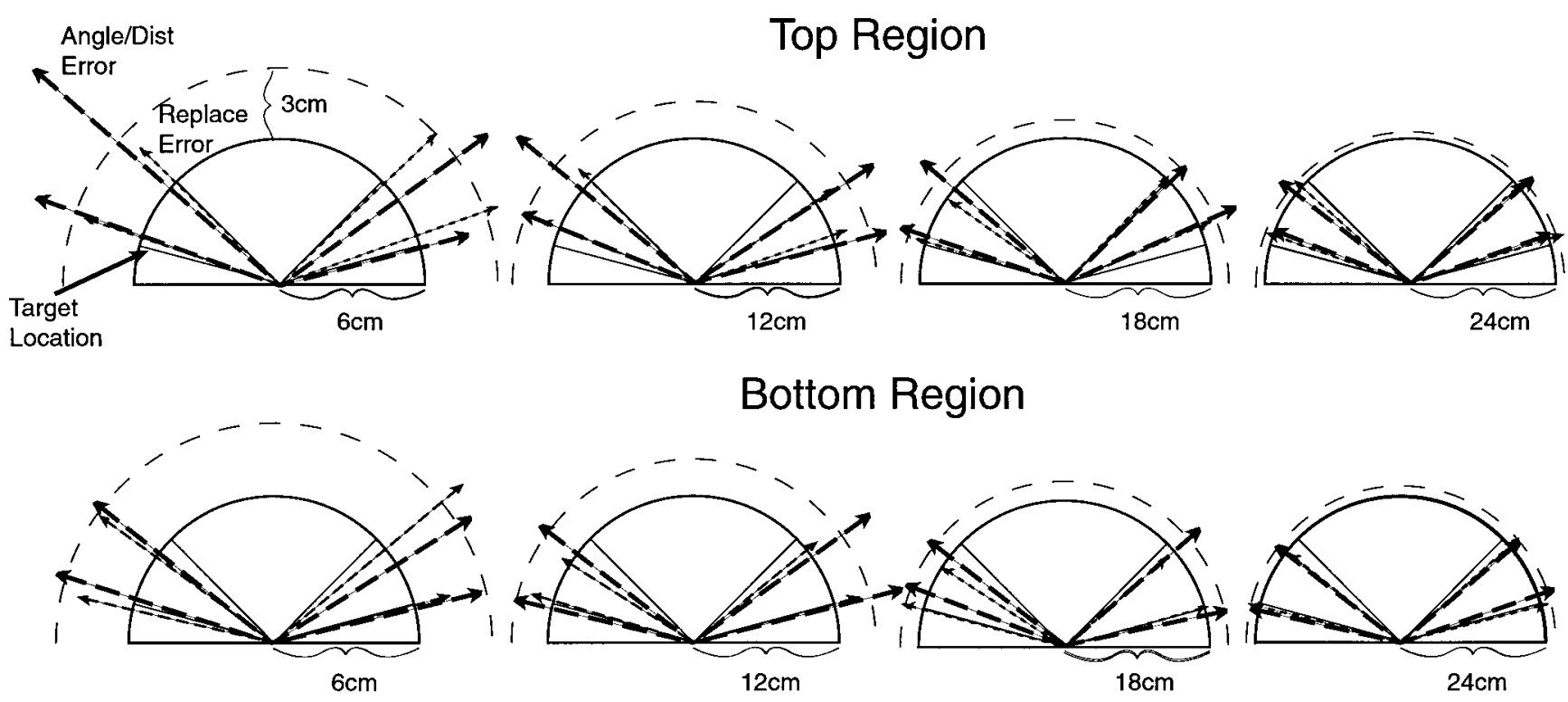

Figure 7. Each semicircular pattern indicates signed errors in Experiment 2 for the four target angles at one region and target distance. The target distance is represented by the solid arc, and the dashed arc represents a distance of $3 \mathrm{~cm}$ from the target. Because the four target distances are all represented by the same-size semicircle, the arc representing $3 \mathrm{~cm}$ from the target indicates the change in scale from one semicircular diagram to another. The target angles are represented by the solid radii. The mean responses are indicated by the arrows; each arrow shows a response distance (within the scale of the particular semicircular pattern, i.e., target distance) and angle. The fine dashed arrow shows these data for the replace instruction, and the coarse dashed arrow combines the angle-estimation and distance-estimation responses, each of which contributed one type of error. Dist $=$ distance.

Table 3

Absolute Error in the Replace and Estimation Instructional Conditions of Experiment 2 by Target Angle and Distance

\begin{tabular}{|c|c|c|c|c|}
\hline \multirow[b]{2}{*}{$\begin{array}{l}\text { Angle and } \\
\text { distance }(\mathrm{cm})\end{array}$} & \multicolumn{2}{|c|}{$\begin{array}{l}\text { Absolute angle } \\
\text { error (deg) }\end{array}$} & \multicolumn{2}{|c|}{$\begin{array}{l}\text { Absolute distance } \\
\text { error }(\mathrm{mm})\end{array}$} \\
\hline & Replace & $\begin{array}{c}\text { Angle } \\
\text { estimation }\end{array}$ & Replace & $\begin{array}{l}\text { Distance } \\
\text { estimation }\end{array}$ \\
\hline \multicolumn{5}{|l|}{$-45^{\circ}$ angle } \\
\hline 6 & 10.2 & 17.5 & 25.3 & 58.5 \\
\hline 12 & 10.3 & 12.5 & 18.6 & 62.3 \\
\hline 18 & 13.7 & 8.6 & 19.0 & 50.3 \\
\hline 24 & 6.8 & 9.9 & 26.4 & 56.9 \\
\hline \multicolumn{5}{|l|}{$-15^{\circ}$ angle } \\
\hline 6 & 14.8 & 12.7 & 28.8 & 43.8 \\
\hline 12 & 7.8 & 10.8 & 19.1 & 32.3 \\
\hline 18 & 4.4 & 6.3 & 28.0 & 37.2 \\
\hline 24 & 3.1 & 6.2 & 30.9 & 29.2 \\
\hline \multicolumn{5}{|l|}{$15^{\circ}$ angle } \\
\hline 6 & 12.2 & 16.3 & 25.7 & 31.0 \\
\hline 12 & 6.8 & 4.6 & 21.8 & 51.1 \\
\hline 18 & 7.1 & 12.0 & 21.7 & 45.7 \\
\hline 24 & 4.9 & 7.8 & 22.0 & 42.9 \\
\hline \multicolumn{5}{|l|}{$45^{\circ}$ angle } \\
\hline 6 & 13.9 & 23.3 & 37.4 & 46.5 \\
\hline 12 & 11.4 & 14.4 & 24.7 & 64.1 \\
\hline 18 & 5.7 & 9.4 & 16.6 & 48.8 \\
\hline 24 & 5.1 & 10.5 & 22.6 & 54.5 \\
\hline
\end{tabular}

Note. $\quad \operatorname{deg}=$ degrees.
$27)=2.99, p<.05$. The latter effect was small and primarily due to one distance.

Signed interfinger distance error decreased with distance and, like absolute error, was consistently higher for the distanceestimation instruction. Thus there were significant main effects of distance, $F(3,27)=7.20, p<.01$, and instruction, $F(1,9)=$ $10.83, p<.01$. The instruction effect was somewhat greater for the wide angles $\left( \pm 45^{\circ}\right)$ and the smaller distances, so that instruction interacted with both angle and distance: $F_{\mathrm{s}}(3,27)=3.72$ and 6.23 , $p$ s $<.01$ and $<.05$, respectively.

Interfinger angle error. The ANOVA on absolute interfinger angle error showed not only significant effects of angle, $F(3,27)=$ $3.50, p<.05$, but also of instruction, $F(1,9)=11.66, p<.01$, and distance, $F(3,27)=14.37, p<.01$. The absolute errors were greater for the more extreme angles and greater by $3^{\circ}$ overall for the angle-estimation instruction than the replace instruction. Finally, as in Experiment 1, there was a tendency for the absolute angle error to decrease as the target distance increased, which can be attributed to measurement artifact.

The ANOVA on signed interfinger angle error included factors of region, angle, distance, and instruction. The only significant effect was that of angle, $F(3,27)=12.68, p<.01$. This reflects the fact that there was a tendency for participants to replicate the large angles $\left( \pm 45^{\circ}\right)$ more horizontally than they actually were and the small angles $\left( \pm 15^{\circ}\right)$ more vertically (as in Experiment 1 , which used only small angles). It is notable that the replace and angle-estimation instructions did not differ significantly in signed error and that there were no interactions involving instruction (all ps $>.15)$. 


\section{Discussion}

The present experiment generally confirmed the results of Experiment 1 with respect to distance errors. Errors in the location of the replaced fingers were of a magnitude comparable to Experiment 1 . The general pattern was for positive signed errors (overestimation of distance), particularly for smaller distances. As before, signed and absolute distance errors were considerably greater when distance was estimated at the front of the table than when the fingers were replaced at the presentation locations. This supports the conclusion from Experiment 1 that a kinesthetic representation of touched locations underlies the relatively accurate responding in the replace condition but that additional computation is needed to report the distance between the fingers at a new location.

Additional findings provided by Experiment 2 pertain to angle error in the estimation and replace instruction conditions, using a wider range of angles than Experiment 1 . Signed errors did not differ between the two instructions, with both conditions showing a tendency for responses to move toward the mean of the target angle distribution. Absolute errors were greater for estimation than replace instructions, but the difference was small: The increase in the estimation error relative to the replace condition was only by about one third, compared with a more than 2:1 increase in distance error across the two responses. Moreover, the difference between instructions was found with absolute but not signed errors, which indicates that the effect lies in nonsystematic error (noise)

On the whole, these results suggest that the metric properties of distance and angle are computed by separate processes that introduce different levels of error. Figure 1 proposes that these processes can be used to form a configural representation. Experiment 3 evaluated this proposal by asking participants to replicate the contacted layout in a new location.

\section{Experiment 3: Distance Estimation Versus Replacing or Translating the Fingers}

In Experiment 2, distance and angle were estimated separately, precluding participants from reproducing the entire configuration of the target locations. Experiment 3 introduced a new "translate" instruction, in which both angle and distance were to be replicated (as in the previous replace condition) but moved to the front of the tablet. The replace and distance-estimation instructions were retained. To the extent that there is a configural representation that combines scale with shape, then distance judgments should be more accurate in the translation condition-in which the entire configuration is replicated - than the condition in which distance is estimated independently of angle. The accuracy of distance estimation in the translation condition would indicate the fidelity of the configural representation with respect to scale. The contribution of a kinesthetic representation may still lead to greater distance accuracy when the fingers are replaced in their original locations than when the configuration is translated.

The predictions about angle reports depend on the efficacy of the configural representation, relative to kinesthesis. Experiment 2 found that replacing the fingers showed no advantage in systematic error (cf. noise) relative to angle estimation in isolation. Presumably, angle estimation by means of the translated configuration would only bring performance closer to the level of accuracy that is found when angles are reported by replacing the fingers at the originally touched locations.

\section{Method}

The participants were 9 male and 3 female university students who received course credit. Two were left handed, and the others were right handed, as indicated by self-report. None had participated in the other experiments reported here.

As before, the participant's index fingers were guided from start locations to two target locations for $3 \mathrm{~s}$, and the fingers were then returned to the start locations. At this position, the experimental instruction was announced as one of the following three types:

Replace: The participant moved the right and left fingers back to their previous locations.

Translate: At the start location, the participant moved the fingers apart and together to replicate the distance between the target locations and vertically to replicate the target angle. He or she could move one finger or both at the same time or separately.

Distance estimation: At the start location, the participant moved the fingers horizontally—simultaneously or separately, right or left—so as to replicate the Euclidean distance formed between the two target locations.

The target pairs fell within a single target region, centered along the sagittal axis of the participant's body, at a distance of $36 \mathrm{~cm}$ from the tablet edge. As in Experiment 2, the target pair members were separated by 6, 12 , 18 , or $24 \mathrm{~cm}$ and formed an angle relative to the horizontal of $-45^{\circ},-15^{\circ}$, $15^{\circ}$, or $5^{\circ}$. The combination of 4 intertarget angles $\times 4$ intertarget distances occurred in a factorial manipulation with the 3 types of instructions for a total of 48 trials. Only one starting location was used-it placed the two fingers $15 \mathrm{~cm}$ apart symmetric about the sagittal plane at a distance of 22 $\mathrm{cm}$ from the tablet edge.

\section{Results}

Location error. This could be measured only for the replace instruction and, as in Experiment 2, showed no effects of distance and angle. The mean error was $26.8 \mathrm{~mm}$, virtually identical to Experiments 1 and 2 (see Table 2).

Interfinger distance error. The effect of instruction on this measure was of principal interest. An instruction effect was confirmed for both absolute and signed error measures: Accuracy was highest for replace instructions, intermediate for translate instructions, and lowest for distance-estimation instructions. Figure 8 shows the error measures by instruction as a function of angle, averaged over distance (angle showed more systematic effects than distance and did not interact with distance). The ANOVA on absolute interfinger distance error showed effects of instruction, $F(2,22)=17.05, p<.01$; angle, $F(3,33)=7.76, p<.01$; distance, $F(3,33)=4.78, p<.05$; and Instruction $\times$ Angle, $F(6$, $66)=4.57, p<.01$. Most important, post hoc Scheffé tests showed that the three instructions all differed significantly from one another. The effects involving distance and angle occurred because absolute interfinger distance error increased with distance and showed a tendency to be greater at the $\pm 45^{\circ}$ angles, especially for the distance estimation instruction.

The ANOVA on signed interfinger distance error showed an effect not only of instruction, $F(2,22)=4.69, p<.05$, but also of 

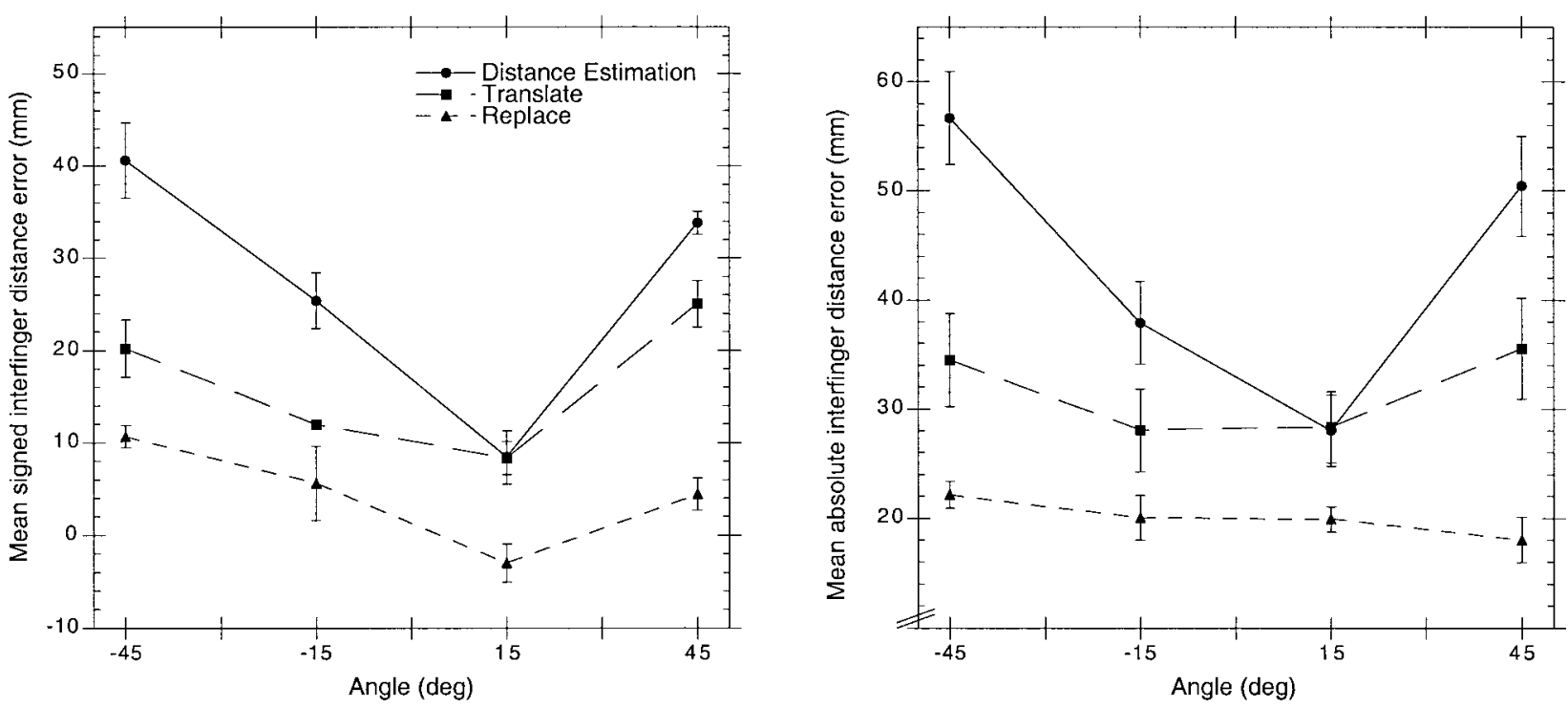

Figure 8. Signed and absolute distance error in Experiment 3 as a function of target angle for each instruction (distance estimation, translate, replace), averaged over distance. Error bars represent standard errors of the means. deg $=$ degrees.

angle, $F(3,33)=12.42, p<.01$. The latter effect was due to higher error for target pairs at the $\pm 45^{\circ}$ angles; other measures (see Experiment 2 results) have previously shown similar effects. Post hoc Scheffé tests showed that the replace and distanceestimation instructions differed significantly from one another and that the translate instruction did not differ significantly from either of the other conditions.

Interfinger angle error. The interfinger angle errors were measured for the replace and translate instructions (see Table 2). The ANOVA on absolute error showed an effect of instruction, $F(1$, $11)=11.12, p<.01$; and distance, $F(3,33)=12.51, p<.01$. Absolute error was greater for the translate than the replace instruction by approximately $3^{\circ}$, and it decreased with distance, which we once again attribute to measurement artifact. The ANOVA on signed interfinger angle error showed an effect of angle, $F(3,33)=14.47, p<.01$, such that the $\pm 15^{\circ}$ angles were drawn toward the vertical, as before. There were also two interactions that were not readily interpretable. One was between angle and distance, $F(9,99)=2.77, p<.01$, and the second was a marginal interaction between instruction and angle, $F(3,33)=$ $2.90, p=.05$. These appeared to reflect modest but consistent variations in systematic trends with stimulus angle. Overall, the instruction effect on signed error was negligible.

\section{Discussion}

The present results point to the use in different tasks of a kinesthetic representation and a configural representation, the latter maintaining scale only to a limited extent. These conclusions are based on comparing the level of distance and angle error across the instructions.

With respect to signed and absolute measures of interfinger distance errors, in terms of accuracy, the conditions were ordered replace, translate, estimation. These results further support the proposal that estimating distance at a new location requires computing the metric property from the kinesthetic representation at a cost in terms of errors. The advantage for the translate condition over estimation indicates that people have a representation of the configuration produced by the fingers on the table top that withstands changes in body position and that maintains scale to some degree, so that it can be used to reproduce the distance between the fingers at a new location in space better than distance can be estimated by itself. However, the level of error in the translate instruction was still substantially greater than was obtained with the replace instruction (see Table 2). Thus the configural representation is not scaled sufficiently to produce an equivalent level of performance to kinesthetic reproduction.

In contrast to performance with distance, angle error showed little difference between replace and translate instructions. On the whole, then, the ability to report angular relations between points in manipulatory space seems minimally vulnerable to a translation of the entire configuration (Experiment 3) or to use of an experimenter-specified distance, which alters configural scale (Experiment 2). The earlier work of Klatzky (1999) suggests, however, that there is a transformation that does affect report of angle-namely, a requirement that the configuration be mentally rotated before responding. This requirement was introduced in Experiment 4 to address whether the representation of shape that supports angle judgments is egocentrically defined.

\section{Experiment 4: Effects of Display Rotation on Distance and Angle Error}

Experiment 4 was motivated by two findings of Klatzky (1999). First, in that study errors in angle and distance were decoupled, in that participants showed virtually no systematic angle error while they were producing substantial systematic error in distance. Similar results were obtained in the present Experiment 2. Second, 
distance and angle errors were differentially affected by imagined transformations between stimulus presentation and response: Distance was affected by imagined translation, and angle by imagined rotation of the display (Klatzky, 1999). The latter result suggests that the configural representation underlying angle errors had an orientation relative to the axes of the body and was not fully object centered. In other words, angles were not defined with respect to intrinsic axes in the configuration itself.

The instructions for angle estimation in Experiments 2 and 3 introduced the frontal axis of the body as a reference axis. This axis is not part of the touched configuration itself, but it forms an implicit component of the layout. If the reference axis that is used to report angle is flexible with respect to an extrinsic frame, angles should be subject to report from any arbitrarily defined value, without an advantage for the frontal axis as a reference. This was tested in the present experiment.

Participants were required in some conditions to rotate the target display in their imagination before reproducing or estimating the distance and angle. We asked whether imagined rotation would particularly affect the angle error, but not the distance error, as had been found previously by Klatzky (1999). The imagined rotation was instituted by having each participant start with his or her index fingers at a given angle relative to one another, move them to a pair of target locations, and then report on the targets from a response position that placed the fingers either at the original start angle or a new angle. Participants were instructed that they should report the positions of the target locations relative to the original start locations, even if the response position differed. When the start and response positions differed, this required a mental rotation. To preface the results, requiring a rotation profoundly impaired angle estimation, although it had no effect on distance estimation.

\section{Method}

Participants. The participants were 8 university students from an introductory psychology class who took part for credit. There were 2 women and 6 men. All reported right handedness. None had participated in the other experiments reported here. Two additional participants were eliminated because of extremely high variability in absolute angle error; these participants accounted for 13 of the 20 highest angle error values, and their inclusion could only inflate the high angle errors reported below under conditions of rotation. The same participants' distance errors, it is worth noting, were near the mean.

Design and procedure. On each trial, three location pairs were defined: start locations, target locations, and response locations. The participant began each trial with the index fingers of the right and left hand at the start locations. From there, the experimenter guided the fingers to the target locations, where they remained for $3 \mathrm{~s}$. From the target locations, the fingers were removed to the response locations, and the participant was to use the response locations to report the distance and angle between the target locations-but relative to the start locations.

The same target layout was used as in Experiment 2; thus, as before, the members of a target location pair were separated by one of four distances-6, 12, 18, or $24 \mathrm{~cm}$ - and formed one of four angles relative to the horizontal: $-45^{\circ},-15^{\circ}, 15^{\circ}$, and $45^{\circ}$. There were two start locations; the points within each were $15 \mathrm{~cm}$ apart and centered on the participant's sagittal plane, with the midpoint of the fingers located $15 \mathrm{~cm}$ inward from the edge of the digital tablet. The angle between the fingers at the start locations relative to the horizontal was either $0^{\circ}$ or $30^{\circ}$. The two possible response locations were coincident with the start locations.

The design involved four variables. One was the orientation of the start location pair: $0^{\circ}$ or $30^{\circ}$. The second was the orientation of the response location pair: $0^{\circ}$ or $30^{\circ}$. Thus on half the trials, the start and response orientation were identical, and on the other half, they differed by a $30^{\circ}$ rotation. When the start and response orientations were at $0^{\circ}$, the condition was the same as the translate instruction of Experiment 3. The remaining variables were the distance and angle formed by the target locations. The factorial combination of the four variables yielded 64 experimental trials, which were preceded by four practice trials to ensure that the participant understood the task

Participants were told that their task was to replicate a target line (defined as being implicit between the target locations) in terms of (a) its length and (b) its angle relative to the start line (defined as being implicit between the start locations). To estimate the angle of the target line relative to the start line, they would begin at a response line (defined analogously). Participants were to move their fingers perpendicular to the response line in either direction until the angle between the start and target lines was replicated, but now using the response line as the base of that angle. Thus, for example, if the start locations were horizontal and the response locations were at $30^{\circ}$, the participant would have to add $30^{\circ}$ to the target angle to make the correct response. They were told that the start line and response line may or may not have the same orientation and that they would not know the orientation of the response line until they responded. To estimate the distance between the target locations, they were to move their fingers together or apart until they were the same distance from one another as the target location pair. Participants could replicate the angle first and then move their fingers to create the distance, or they could do the reverse; they were also free to move one finger or both at the same time or separately. The experimenter demonstrated these responses with examples, including some in which the start and response orientations differed, until the participant indicated that he or she understood the task of replicating the relative orientation between the start and target lines at the response line, as well as the distance between the target locations.

\section{Results}

Due to experimenter error, one missing data point for 1 participant was replaced with the mean for all others. In addition, six observations yielding absolute angle errors greater than $100^{\circ}$, and three observations yielding absolute distance errors greater than $100 \mathrm{~mm}$ were replaced with the value of 100 . The summary in Table 2 reports errors for the condition in which the start and response lines were horizontal (i.e., the $0 / 0$ condition, comparable with translate instructions in Experiment 3).

Interfinger angle error. The ANOVAs incorporated four factors: start location orientation, response location orientation, target angle, and target distance. Figure 9 shows signed angle error as a function of start and response orientation and target angle, averaged over distance. The ANOVA on signed angle error showed effects of start orientation, $F(1,7)=22.99, p<.01$; response orientation, $F(1,7)=29.38, p<.01$; and target angle, $F(3,21)=$ $3.35, p<.05$. When the start and response orientation were $0^{\circ}$, there was a tendency for the $\pm 15^{\circ}$ angles to be moved more toward the vertical, as has been found consistently across these studies. A start orientation of $30^{\circ}$ and response of $0^{\circ}$ produced more positive error, whereas the combination of $0^{\circ}$ start and $30^{\circ}$ response orientation produced negative error. This indicates that participants, particularly when the start and response orientation mismatched, which required them to output a rotation of the target configuration, underrotated, in effect moving their output closer to the configuration they had experienced. As the two types of mismatches canceled, average signed error was low in the mismatching conditions $\left(3.0^{\circ}\right)$, as well as in the matching conditions 


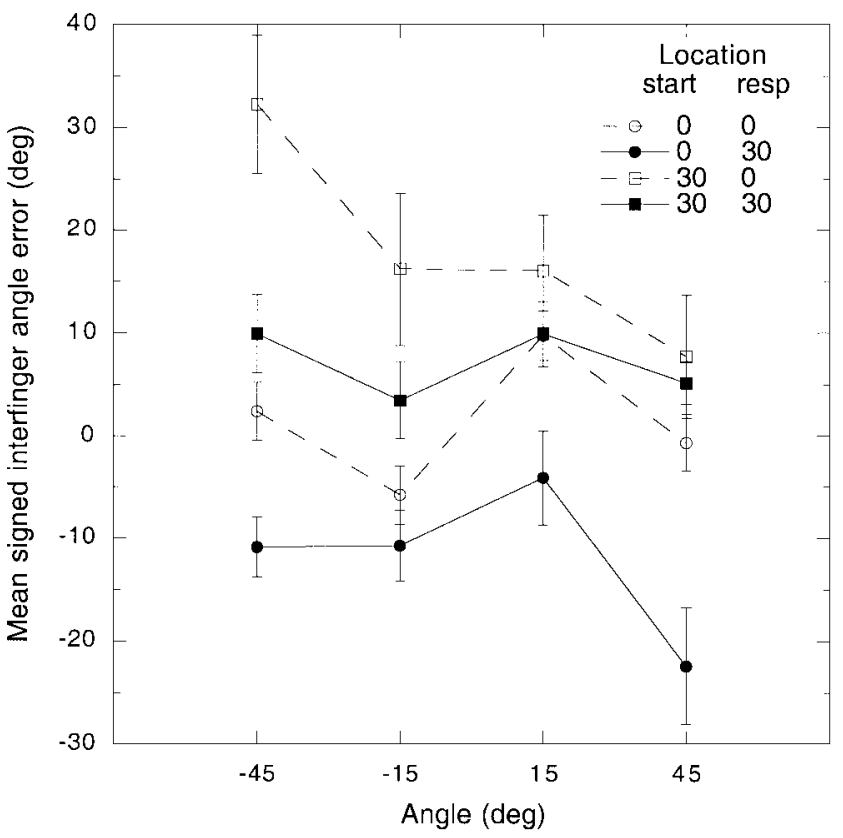

Figure 9. Signed angle error in Experiment 4 as a function of target angle for each combination of start and response (resp) locations (defined by orientation in degrees [deg]), averaged over target distance. Error bars represent standard errors of the means.

$\left(4.3^{\circ}\right)$. The Start Orientation $\times$ Response Orientation interaction did not approach significance in signed error $(F<1)$.

The effect of the match between start and response orientations was shown, however, in the ANOVA on absolute angle error, which had only one significant effect: the Start Orientation $\times$ Response Orientation interaction, $F(1,7)=28.85, p<.01$. Figure 10 shows absolute angle and distance errors together, as a function of the start and response orientation. The absolute angle error averaged $12.9^{\circ}$ in the matching conditions ( $\mathrm{cf} .7^{\circ}$ and $10^{\circ}$ in the previous experiments) and $22.6^{\circ}$ in the mismatching conditions, constituting almost a doubling of error due to the imagined rotation.

Interfinger distance error. As shown in Figure 10, in contrast to the effects on target angle, an imagined rotation before the response had no effect on participants' ability to report the target distance. The analysis of absolute distance error revealed no effects that approached significance. In particular, neither signed nor absolute distance error showed an effect of the match between the start and response orientations (the interaction produced an $F<1$ in both ANOVAs). Over the four combinations of start and response orientations, the signed distance error varied only from 12.8-16.7 $\mathrm{mm}$, and the absolute distance error varied only from 27.3-28.7 mm. The ANOVA on signed distance error did show effects of target angle, $F(3,21)=4.89, p<.01$, and distance, $F(3$, $21)=3.48, p<.05$. Generally, signed distance error went from overestimation to near zero as distance increased, and the more extreme target angles led to increased signed distance error.

\section{Discussion}

The requirement that the display be mentally rotated between presentation and test produced a substantial increase in angle error but did not affect distance. This result parallels that of Klatzky (1999) and supports the idea that different processes are used to abstract angle and distance from a haptic display. We have argued that angle, in particular, makes use of a configural representation of the shape of the touched layout that does not accurately convey scale. These results indicate further that the configural representation is tied to a fixed extrinsic frame of reference; thus, a rotation substantially impairs its usefulness.

\section{General Discussion}

The assumption of the present research is that while touching a sparse set of locations without vision, participants form one or more types of representation from which they attempt to derive answers to spatial questions about the contacted points. As we noted in the introduction, distinctions have been made between various representations of spatial location. The present experiments are consistent with the idea that multiple representations are operative, depending on instructions in the task at hand. They further support our fundamental assumption that derivation of successive representations introduces error. A kinesthetic representation appears to retain location information, thus supporting accurate returns to previously touched locations. From this, reports of distance and angle as abstracted parameters must be computed These appear to be mediated by an oriented, and to some extent scaled, representation of the configuration of the contact points that is not tied to a particular location in space.

Experiment 1 demonstrated the efficacy of the kinesthetic representation for reproducing the locations of the two index fingers. Participants could replace their two fingers in previously felt positions within $3 \mathrm{~cm}$ on average, and the error varied little with interfinger distance. That the underlying representation was kinesthetic is indicated, in part, by its dependence on limb configuration: There was a pattern of increasing error across conditions in

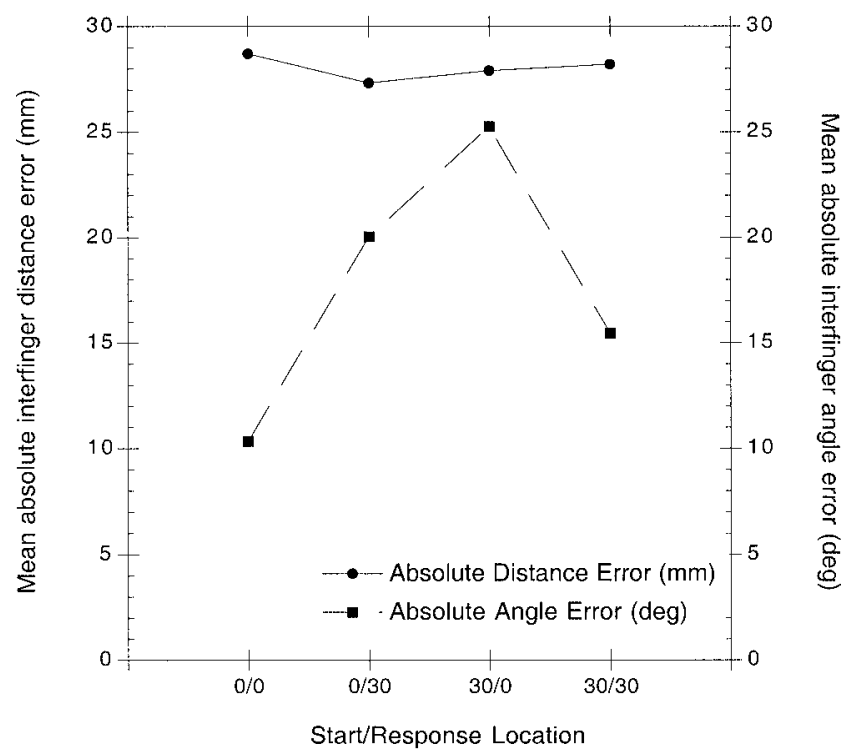

Figure 10. Absolute angle error and absolute distance error in Experiment 4 as a function of start and response locations (defined by orientation in degrees [deg]). Error bars represent standard errors of the means. 
which participants (a) replaced both fingers, (b) replaced one finger only, or (c) exchanged the responding finger between exposure and test.

Further findings indicate the cost of computing distance as a metric property of the touched layout. To estimate distance without replicating contact positions, participants separated the fingers horizontally at a new location. There was consistently found to be far greater interfinger distance error for estimation (without translation of the whole configuration) than replacement. This distance estimation was strongly dependent on the actual distance and to some extent on the response region (Experiment 1).

Experiment 3 directly probed for a nonkinesthetic form of representation that depicted the configuration of the touched locations. Participants either replaced their fingers, estimated distance alone in a new location, or translated the target distance and angle to the new location. Distance responses were still most accurate when participants replaced their fingers at the targets, indicating the advantage of kinesthetic memory. However, the translated distance, where the angle between the fingers was replicated as well, was more accurate than distance estimation along the horizontal axis. The advantage for translation indicates that there is a representation of the target configuration that is not tied to its absolute location in space and that incorporates scale along with shape.

Several findings suggest, however, that the representation of the touched configuration derives from more accurate computation of shape than scale and therefore is more useful for producing angle responses than distance. (a) The relatively large increase in distance error when participants translated the configuration, relative to when they replaced the fingers (Experiment 3), suggests that the kinesthetic representation maintains the scale of the touched layout far better than the configural representation. (b) In contrast, the relatively small difference in angle error between the corresponding translate and replace conditions (only $3^{\circ}$ in absolute error and negligible in signed error in Experiment 3 ) indicates that shape can be derived accurately from kinesthetic input, despite lack of precision in scale. (c) The comparably small difference in angle error between replacing the fingers and estimating angle in Experiment 2 further indicates that shape can be extracted independently of scale and spatial location.

The absence of a precise representation of scale may underlie people's demonstrated tendency to take more ad hoc, heuristic approaches to distance estimation. Often, these heuristics appear to be based on memory for movement, as was found by studies reviewed in the introduction. Given the requirement to report isolated parameters of a haptic display, apparently people use any available representations, together with imposed analytic or heuristic processes. The result when the distance between contacted locations is reported is systematic error and noise. In contrast, as long as a person's orientation relative to the display is maintained, there appears to be consistent and, on average, unbiased report of angular parameters of touched layouts.

As for the orientation of the configural representation, evidence for its being defined relative to an extrinsic reference frame was demonstrated in Experiment 4. Rotating the layout relative to the response generated absolute angular errors of $20^{\circ}-25^{\circ}$-nearly double the average unrotated values. The reliance of angle reports on maintaining the original orientation indicates that the represen- tation is tied to the axes of the body or extrinsic axes (e.g., the table edge). Thus, this result indicates an interaction between extrinsic and configural representations, as is outlined in Figure 1.

A number of research issues present themselves on the basis of the present body of work. As a methodological consideration, the present tasks began with the experimenter's transporting the participant's hand to the contact locations; that is, the tasks involved passive exposure. This method is in keeping with our interest in representations of terminal location rather than movement per se; moreover, active control would necessitate having the participant monitor additional positioning cues. There is some evidence that passive control eliminates cues associated with voluntary movement that can guide position perception (e.g., Laufer, Hocherman, \& Dickstein, 2001; Paillard \& Brouchon, 1968; see also Smyth, 1984, regarding active superiority in movement reproduction). Passive control is not inevitably inferior, however. Sittig, Denier van der Gon, and Gielen (1985) found equivalent performance in visually perceiving the position of a passive arm and performance of slow active position matching; Sittig, Denier van der Gon, and Gielen (1987) later suggested that rather than active movement being a cue to position, slow arm movements are actually dependent on afferent positional cues. It would be of interest to perform similar experiments with active movement, but there seems little reason a priori to assume that the present results depend on passive arm transport.

More detailed kinematic data and response times would also be illuminating in the present tasks. This would enable us to compare the latency for initiating estimation and replacement responses and to determine the similarity between limb positions between exposure and replacement. As we mentioned above, it is likely that the kinesthetic trace is of limited duration. To determine the time course of this trace, another useful manipulation would be to delay the replacement response and examine the effect on error. A further question that could be addressed is whether having tangible markers at the contact points would affect performance, as opposed to the uniform surface now used. Previous findings indicating that cutaneous stimulation affects pointing error (Rao \& Gordon, 2001) make this an intriguing possibility.

Finally, our results have implications for the use of haptic interface devices that use force feedback to guide learning of complex hand movements. The user can be constrained by force fields (e.g., Solis et al., 2002) or can be passively guided, as in the studies described here. Our results suggest that passive guidance would be more effective in teaching the shape of a target movement than its absolute scale. Ancillary cues to scale could be necessary for successful learning. In a relevant study, Feygin, Keehner, and Tendick (2002) asked participants to hold a manipulandum while the device guided the hand through a 3-D sinusoidal motion, simulating surgical threading. The authors found that relative to a visual control, haptic guidance particularly aided learning of the movement's timing. The ability to encode movement timing may help to explain why people often default to movement-based heuristics when asked to report distance between the fingers. More generally, when people attempt to learn sizespecific shapes from sparse kinesthetic inputs, temporally based heuristics may be a means of compensating for the deficiencies in coding scale that are documented here. 


\section{References}

Armstrong, L., \& Marks, L. E. (1999). Haptic perception of linear extent. Perception \& Psychophysics, 61, 1211-1226.

Baud-Bovy, G., \& Viviani, P. (1998). Pointing to kinesthetic targets in space. Journal of Neuroscience, 18, 1528-1545.

Brambring, M. (1976). The structure of haptic space in the blind and sighted. Psychological Research, 38, 283-302.

Fasse, E. D., Hogan, N., Kay, B. A., \& Mussa-Ivaldi, F. A. (2000). Haptic interaction with virtual objects: Spatial perception and motor control. Biological Cybernetics, 82, 9-83.

Feygin, D., Keehner, M., \& Tendick, F. (2002). Haptic guidance: Experimental evaluation of a haptic training method for a perceptual motor skill. In Proceedings of the 10th Symposium on Haptic Interfaces for Virtual Environment and Teleoperator Systems (pp. 40-47). Los Alamitos, CA: IEEE Computer Society.

Helms-Tillery, S. I., Flanders, M., \& Soechting, J. F. (1994). Errors in kinesthetic transformations for hand apposition. NeuroReport, 6, 177181

Huttenlocher, J., Hedges, L. V., \& Duncan, S. (1991). Categories and particulars: Prototype effects in estimating spatial location. Psychological Review, 98, 352-376.

Jaric, S., Corcos, D. M., Gottlieb, G. L., Ilic, D. B., \& Latash, M. L. (1994). The effects of practice on movement distance and final position reproduction-Implications for the equilibrium-point control of movements. Experimental Brain Research, 100, 353-359.

Klatzky, R. L. (1998). Allocentric and egocentric spatial representations: Definitions, distinctions, and interconnections. In C. Freksa, C. Habel, \& K. F. Wender (Eds.), Spatial cognition-An interdisciplinary approach to representation and processing of spatial knowledge (Lecture Notes in Artificial Intelligence 1404; pp. 1-17). Berlin, Germany: SpringerVerlag.

Klatzky, R. L. (1999). Path completion after haptic exploration without vision: Implications for haptic spatial representations. Perception \& Psychophysics, 61, 220-235.

Larish, D., \& Stelmach, G. E. (1982). Spatial orientation of a limb using egocentric reference points. Perception \& Psychophysics, 32, 19-26.

Laufer, Y., Hocherman, S., \& Dickstein, R. (2001). Accuracy of reproducing hand position when using active compared with passive movement. Physiotherapy Research International, 6, 65-75.

Lederman, S. J., Klatzky, R. L., \& Barber, P. (1985). Spatial- and movement-based heuristics for encoding pattern information through touch. Journal of Experimental Psychology: General, 114, 33-49.

Lederman, S. J., Klatzky, R. L., Collins, R., \& Wardell, J. (1987). Exploring environments by hand and foot: Time-based heuristics for encoding distance in movement space. Journal of Experimental Psychology: Learning, Memory, and Cognition, 13, 606-614.

Lederman, S. J., \& Taylor, M. M. (1969). Perception of interpolated position and orientation by vision and active touch. Perception \& Psychophysics, 6, 153-159.
Loomis, J. M., Da Silva, J. A., Fujita, N., \& Fukusima, S. S. (1992). Visual space perception and visually directed action. Journal of Experimental Psychology: Human Perception and Performance, 18, 906-921.

Loomis, J. M., \& Philbeck, J. W. (1994, November). Distortion of visual space under full cues is not scale-invariant. Paper presented at the 35th Annual Meeting of the Psychonomic Society, St. Louis, MO.

Millar, S. (1976). Spatial representation by blind and sighted children. Journal of Experimental Child Psychology, 21, 460-479.

Millar, S. (1994). Understanding and representing space: Theory and evidence from studies with blind and sighted children. New York: Oxford University Press.

O'Malley, M. K., \& Goldfarb, M. (2002). Comparison of human haptic size identification and discrimination performance in real and simulated environments. In Proceedings of the 10th Symposium on Haptic Interfaces for Virtual Environment and Teleoperator Systems (pp. 10-17). Los Alamitos, CA: IEEE Computer Society.

Paillard, J., \& Brouchon, M. (1968). Active and passive movements in the calibration of position sense. In S. J. Freedman (Ed.), The neuropsychology of spatially oriented behavior (pp. 37-55). Homewood, IL: Dorsey Press.

Rao, A. K., \& Gordon, A. M. (2001). Contribution of tactile information to accuracy in pointing movements. Experimental Brain Research, 138, $438-445$.

Rosenbaum, D. A., Meulenbroek, R. G., \& Vaughan, J. (1999). Remembered positions: Stored locations or stored postures? Experimental Brain Research, 124, 503-512.

Sittig, A. C., Denier van der Gon, J. J., \& Gielen, C. C. A. M. (1985). Separate control of arm position and velocity demonstrated by vibration of muscle tendon in man. Experimental Brain Research, 60, 445-453.

Sittig, A. C., Denier van der Gon, J. J., \& Gielen, C. C. A. M. (1987). The contribution of afferent information on position and velocity to the control of slow and fast human forearm movements. Experimental Brain Research, 67, 33-40.

Smyth, M. M. (1984). Memory for movements. In M. M. Smyth \& A. M Wing (Eds.), The psychology of human movement (pp. 83-117). San Diego, CA: Academic Press.

Solis, J., Avizzano, C. A., \& Bergamasco, M. (2002). Teaching to write Japanese characters using a haptic interface. In Proceedings of the 10th Symposium on Haptic Interfaces for Virtual Environment and Teleoperator Systems (pp. 255-262). Los Alamitos, CA: IEEE Computer Society.

van Beers, R. J., Sittig, A. C., \& Denier van der Gon, J. J. (1998). The precision of proprioceptive position sense. Experimental Brain Research, 122, 367-377.

Wallace, S. A. (1977). The coding of location: A test of the target hypothesis. Journal of Motor Behavior, 9, 157-169.

Received September 20, 2001

Revision received April 18, 2002

Accepted July 29, 2002 